\title{
Research on the Protection and Restoration of Historical Blocks Based on Landscape Cultural Ecology
}

\author{
Li Yan \\ Huanghe Science and Technology College \\ Zhengzhou, China
}

\begin{abstract}
When facing the same fact or event, because of the differences on the standpoint and background of "seeing" and "thinking", there may be conflicts and divergences in "doing". There is no exception for the protection of historical blocks. Because of this, many problems emerge in the protection and restoration of historical blocks from the traditional perspective: emphasize economy and belittle culture; emphasize material and belittle non-material; emphasize drama effect and belittle historical accumulation; etc.. Could it be said that the protection and restoration of historical blocks only is about the reservation of the blocks size and the architectural form, and obtain enormous economic benefits? No, the value of historical blocks should be a multi-valued complex. Therefore, it is necessary to illustrate the protection and restoration of historical blocks from a more macroscopical and systematic perspective. Based on this, this thesis aims to provide some new view, new standpoint and new standard for the protection and restoration of historical blocks by introducing landscape cultural ecology.
\end{abstract}

Keywords-landscape cultural ecology; historical blocks; protection and restoration

\section{INTRODUCTION}

The protection and restoration of historical blocks in China had gone through the experience from "pull down and rebuild" to "protection and restoration", from being left without anybody to care for in ruins to very popular, which is a great leap. However, the protection and restoration of historical blocks have not received the desired effect dut to the lack of recognition and deviate protection concept. Generally, the reasons can be classified into the following three kinds.

\section{A. Emphasize Economy and Belittle Culture}

In the 1990s, the rapid developing economy made the modern social value tend to be "interests above everything else"; especially the unification of products and simplification of value orientation brought by industrialization made individuality cannot be extended. People were more satisfied with the accumulation of tangible assets, but only required instant satisfaction towards culture, the intangible asset of the city. Under the background that material condition became the primary requirement and spiritual demand was the secondary requirement, culture carried by historical blocks was abandoned without reluctance.

\section{B. Emphasize Material and Belittle Non-Material}

The formation of the environment of historical blocks has undergone a very long process. Environment here refers to not only the material environment, but also the overall environment composed by residents of the blocks, historical buildings and surrounding environment. That is to say, environment of historical blocks includes the material environment of human landscape, static natural environment and dynamic living environment, which are the complete overlaying of the mutual influence and effect of all kinds of environment. People may doubt that many historical cities have followed their original style and features without any protection during hundreds and thousands of years. In contrast, the recent decades' protection work has no lasting appeal. One reason that cannot be ignored is that historical environment has not been completely protected.

\section{Emphasize Drama Effect and Belittle Historical Accumulation}

No matter the planner, original residents in the historical blocks, or the government worker, they may ask unconsciously during the protection process: what kind of history shall we protect? This not only refers to the authenticity of the protection, but also directly affects the appearance of the blocks after restored. However, the planning and design of many historical blocks equal the "protection and restoration" to the rebuilding of common tourism interests and old blocks, leading to many wrong practices. For example, set all the architecture into a certain period subjectively for the sake of protecting "complete historical appearance"; seal the historical blocks for safekeeping without considering the requirements of modern lives.

\section{REASON OF INTRODUCING LANDSCAPE CULTURAL ECOLOGY}

Why does the protection of historical blocks always being defeated when confronted with economic benefits? Why does material environment and non-material environment cannot be equally treated in the protection process of historical blocks? Why does the historical accumulation of different times cannot be fairly considered in the protection of historical blocks? These are the questions worth thinking. One of the important 
reasons is that the culture of historical blocks has not being correctly recognized. Culture in the eyes of historian, sociologist, and architect and city planner is more of academic value; culture in the eyes of developers is the economic value brought by it; culture in the eyes of residents is the improvement of material condition brought by it. In fact, there is no conflict among the different values from different view. Historical blocks are the multi-value complex essentially. However, discuss the protection and restoration of historical blocks only from one aspect would cause imbalance of cultural development.

Historical blocks are complex systems to demonstrate different characteristics of society, economy, geology and people and so on. The cultural development of historical blocks has close relationship with the concrete environment, natural condition, economy, technology and history. Material and non-material culture and environment and people living in combine the living culture, which is growing and changing continuously. It is isolated and one-sided to consider culture as it's simply culture, or discuss material cultural relics protection only for the protection. Therefore, it is necessary to introduce landscape cultural ecology to study the protection and restoration of historical blocks from a more macroscopical and systematic perspective. As a comprehensive subject guiding cultural construction, landscape cultural ecology certainly can provide new view and method for the protection and restoration of historical blocks. The author summarizes the following three points.

- The introduction of landscape cultural ecology helps correctly recognize the culture of historical blocks. There are many misunderstandings towards culture in the present protection and restoration work, such as simply treat historical culture as the material remains. Landscape cultural ecology is science of socialcultural studies that combines all kinds of complicated cultural factors and illustrates the culture's composition and evolution, and thus provides guidance to the determination of protection contents for historical blocks.

- The introduction of landscape cultural ecology helps scientifically guide the protection and restoration of historical blocks. According to landscape cultural ecology, all culture processes in history are the processes of ecological balance. The relationship between different cultures and the relationship among culture, environment and people is mutual influential, mutual promotion and mutual restrictive. Therefore, the cultural ecology of historical blocks is surely a dynamic system. It requires that the previous static protection mode in the protection and restoration of historical blocks should be changed and the observation and study historical culture and material relics should be integrated into the interactive relationship between human and environment.

- The introduction of landscape cultural ecology helps change the value of "economy supremacy" into "culture orientation" value, and transform from single value to multi value in the protection and restoration of historical blocks. According to landscape cultural ecology, the protection and restoration of historical blocks not only is about the development of economy, but also cover the continue of historical context.

\section{ANALYSIS ON THE VALUE OF INTRODUCTION}

What are the values by introducing landscape cultural ecology? How to illustrate the existing problems in the present protection and restoration work? In the process of interpreting the problems and puzzles of protection and restoration work from the perspective of landscape cultural ecology, the author draws out three values by introducing landscape cultural ecology in protection and restoration work.

\section{A. Harmonious Co-Prosperity of Historical Culture and Economic Development}

Landscape cultural ecology takes culture as the main line and studies on contents like culture mode, culture transition and cultural inheritance, etc.. It introduces the theory and research method of ecology into culture research, forming the holistic approach and broad perspective to observe nature, science and technology, economy, society, culture and related factors in a systematic way. Similar with maintaining the ecological balance of nature in ecology, cultural ecology emphasizes the harmonious co-prosperity among all elements within the system. It considers historical blocks as a complete system. All variables like cultural inheritance, economic development, and social life and so on are promoted by each other, influenced by each other and restricted by each other. Excessive emphasis on one of the aspects would lead to the imbalance of the whole system. Only through the good coordination of the relationship among all variables can the whole system achieve the sustainable development.

That is to say, the value of historical blocks can be maintained in a stable and balanced condition only through the realization of the values of all elements within the system. Compared with entity elements in a city, it has stronger comprehensiveness. For example, the value of cultural relic historical architecture and architectural complex is more centered on the historical cultural value, which is incalculable. The economic value is always estimated by converting the cultural value under different conditions. The value of other city entities that are non-historical blocks is always represented on the economic utilization, and cultural value always give way to economic value.

Historical blocks not only have certain historical cultural value, but also possess considerable economic value. Only the two combine properly and rely on each other, historical blocks can be able to receive considerable development. Only emphasize cultural value and give up the due economic value, or realize considerably high economic value but destroy the cultural value would all directly lead to the decline of historical blocks. In the protection and restoration of historical blocks, cultural protection and economic development is a "knot", but not a "dead knot". The protection and restoration of historical blocks can achieve the culture sustainment and lasting economic prosperity by relying on historical cultural value and backed by economic value. 


\section{B. Equal Treatment on Material and Non-Material Environment}

Landscape cultural ecology conducts cultural research through a comprehensive and systematic theory and approach, and starts from the balanced relation of nature, human and society. It takes emphasis on both the creation of tangible and natural material culture and the creation of intangible, nonmaterial culture.

Meanwhile, it introduces "environmental perspective" into the research of culture. Similar with natural environment taking the core position in natural ecology, cultural environment also takes the core position in cultural ecology. Culture can only survive under certain environment, which includes material environment, as well as non-material environment. Some culture types are influenced by material environment as the products of material environment, but also influenced by culture themselves being not the single products of material environment. Material environment affects the cultural creation. In the meantime, non- material environment, including religious belief, value, morality and ethics, manners and customs and so on, also influences the existence and development of culture. They are influenced and restricted by each other.

Thus it can be seen that culture beard by historical blocks can only exist in the environment it was born form. Once losing the oiling of environment, the dialogue between historical blocks and modern cities would be uninteresting and farfetched, and the expressive power of historical culture would also be damaged. Environment here refers to not only the material environment composed of architecture, courtyard, and blocks, but also the non-material environment composed of life style, neighborly relations, folk custom, ideology, religious belief and so on.

The protection and restoration of historical blocks under the traditional perspective often took material environment and non-material environment under unequal position, more emphasized on the reconstruction of material environment but neglect the protection of non-material environment like folk custom, life style, and ideology, etc...

The introduction of landscape ecology emphasizes the equal treatment towards material and non-material environment, not only emphasize the improvement of the physical and chemical environment within the blocks, but also pay more attention on the deduction of the humanistic spirit based on physical and chemical environment and the respect and treasure on the overall historical environment. Therefore, the reconstructed historical blocks appear in front of people in an overall image that consists of center, blocks, boundaries, smell of everyday life and soul of life, rather than a single renovated cultural relic building or fragmented historical memories.

Therefore, view the protection and restoration of historical blocks from the perspective of landscape cultural ecology is considering the existing and development of blocks from the integrated environment view. It has changed the single environment view of repairing blocks' material environment only under the traditional perspective and combine non- material environment into it, forming a completely new recognition of people toward "complete historical style" and guiding the extension of the protection contents of historical blocks, and thus realizing the sustainable development of historical blocks much better.

\section{Harmonious Coexistence of the Historical Accumulation of Different Periods}

According to landscape cultural ecology, the overall cultural ecosystem consists of space, culture and human, which are influenced, promoted and restricted by each other. Accordingly, landscape cultural ecology must be a dynamic system. It is wrong to consider it as a static system or freezeframed in a certain fragment. Culture must be taken into observation and research into the interaction with environment and human, so as to truly understand the development and transformation of culture.

Since the come into being, historical blocks had undergone a dynamic changing process. It not only recorded all the information of historical culture during different periods, but also interpreted the real life and events that will occur in the future continuously. The material remains of historical blocks are the material reflection of history. The addition and revision parts of remains reflected the evolution and change of historical life and the living condition of people during different periods.

The material remains of historical blocks are composed by blocks and roads including street, lanes and alleys, communities and neighbors consisting of courts and yards and courtyards constituted by architectures and buildings. These material relics were all constructed around our ancestors' living demands and their understanding towards fashion at a specific time. From the dynamic perspective we can know that material remains have reflected people's understanding towards life after several adding and changing since its creation, showing the information of historical cultural changes. The changing of material remains and the evolution of spiritual culture were basically synchronous. It was because of the highly unity of the material life and spiritual life of our ancestors that we can feel the development process of historical blocks and the historical accumulation of all periods.

Any historical blocks were not made instantly. Therefore, there was no unity on the style of historical blocks. The protection concept under traditional perspective treated history in a static opinion, choosing some angles to conduct positioned protection. This method can be used in making movies. However, historical blocks are living materials, which should reflect the historical accumulation of all periods and satisfy the needs in real life.

\section{CONCLUSION}

The introduction of landscape cultural ecology actually made us consider the development of historical blocks in a dynamic view. Emphasize the historical accumulation of all times, treat history equally, oppose "freeze-frame" in any form, oppose "fragment" in any form, and oppose any protection based on "political demand", and thus make historical blocks 
become the living evidence of life changes history and social development history.

\section{REFERENCES}

[1] Ruan Yisan. Misunderstanding and Misreading of Historical Blocks Protection. Planner.1999, (04): 21-22.

[2] Feng Jicai. Rescue Old Blocks. Beijing: Xiyuan Publisher, 2002, 37-39.

[3] Feng Tianyu. Outline of Cultural Ecology, Knowledge Engineering, 1990, (4): 13-14.

[4] Shan Jixiang. From "Function City" to "Cultural City" [M]. Tianjin University Press, 2007. 